\title{
UNA PERSPECTIVA DE LOS SERVICIOS SOCIALES EN ESPAÑA
}

\author{
M. ${ }^{a}$ DEL CARMEN ALEMAN BRACHO \\ Catedrática de Servicios Sociales de la EUTS \\ de la Universidad de Granada
}

RESUMEN

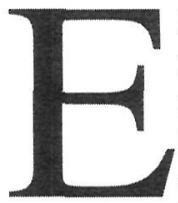

1 artículo comienza refiriéndose a los cuatro sistemas o formas de hacer frente a las necesidades como son los sistemas privados aislados, sistemas de organizaciones voluntarias de acción social, sistema comercial y el sistema público. Continúa luego sintetizando los antecedentes de los Servicios Sociales, hasta llegar a la situación actual, planteando las normas básicas que los regulan, cómo están estructurados, las áreas o sectores donde actúan y cómo son financiados. Por último, termina con unas consideraciones generales sobre los problemas que afronta este sector de tan gran expansión en España desde el advenimiento de la democracia.

\section{INTRODUCCION}

En el análisis de los Servicios Sociales en España, importa destacar, de entrada, que existen diversos modos de hacer frente a las necesidades sociales. Existen, en efecto, distintos sistemas de prestación de asistencia a las necesidades sociales. La Iglesia y el Estado han tenido el protagonismo, pero no la exclusividad. Junto a la Iglesia y al Estado han existido otras modalidades de atención (1).

En primer lugar, los sistemas privados aislados, conocidos como sistema espontáneo de ayuda o Informal. Realizado por familia, amigos o vecinos, es el sistema más antiguo, pero no ha desaparecido. Prueba de ello es la obligación legal de prestación de alimentos entre parientes, establecida desde antiguo en el Derecho civil y, que aún subsiste, entre otras muchas formas de apoyo espontáneo entre particulares.

Una segunda modalidad es el sistema de organizaciones privadas de acción social. Se trata de organizaciones voluntarias sin ánimo de lucro, que actúan dentro de un marco organizado y con una finalidad 
social. Pueden revestir múltiples modalidades, por ejemplo fundaciones, legados, patronatos, así como organizaciones no gubernamentales. Cáritas y la Cruz Roja, son los ejemplos más conocidos. Estas organizaciones han tenido siempre relevancia y continúan teniéndola. Son consideradas como colaboradoras de los poderes públicos, recibiendo ayudas estatales en forma de subvenciones o desgravaciones fiscales.

En tercer lugar, los sistemas comerciales. Consisten en una modalidad de asistencia que se presta mediante organizaciones privadas y empresas de Servicios, pero con fin lucrativo. Es un sistema frecuente en la cobertura de determinados Servicios, por ejemplo: Residencias de ancianos, guarderías, etc. Este sistema no debe descartarse que incremente su frecuencia en el futuro.

La respuesta estatal o pública a las necesidades, es la que asume el protagonismo en la actualidad. El papel estatal es en nuestros días tan acusado que incluso realiza el control, verificación o inspección de los anteriores sistemas, como se verá a continuación.

\section{EVOLUCION HISTORICA DE LA ACCION SOCIAL}

Centrándonos en la evolución histórica de la acción social, se pueden distinguir diferentes fases o etapas. Se trata de fases sucesivas, pero debe subrayarse que la aparición de cada una no elimina por completo a la anterior. Coexisten en cada período (2).

La primera fase está basada en la caridad, es la más antigua, se desarrolla fundamentalmente hasta la Edad Media, con una raíz religiosa. En gran parte las situaciones se abordaban intentando reducir sus manifestaciones más extremas, pero nunca abordando las causas que la ocasionaban. Esta situacion se ponía de manifiesto, por ejemplo, en la legislación; la Novísima Recopilación o las Partidas, «que establecen que los Reyes, clero y prelados, tienen la obligación de atender a los pobres que acudan a ellos».

La segunda etapa de la acción social es la beneficencia pública. Se trata de una organización o actividad que se concreta en la realización de prestaciones graciables, de mera subsistencia, en favor de los necesitados, financiada con fondos públicos y privados. Un rasgo esencial de la beneficencia es que no genera derechos; no existe derecho a obtener la prestacion (3).

La beneficencia conlleva una importante novedad: es que aparece ya la intervención pública, todavía escasa, para hacer frente a las necesidades.

Los inicios de la beneficencia pública se pueden situar en el siglo XVI, con el humanismo del Renacimiento, que centra su preocupación por el hombre y su suerte en este mundo, frente a la preocupación por el otro que prevalecía en la sociedad medieval. 
En esta epoca surge, precisamente, la polémica sobre la pobreza. Las aportaciones de tratadistas como Juan Luis Vives, Domingo de Soto o Cristóbal Pérez de Herrera, y tantos otros, abordan ya los temas centrales para la aparición de la beneficencia pública. Su influencia fue decisiva en los siglos sucesivos.

El siglo XVII parece una continuidad del anterior, pero se están fraguando grandes cambios. El poder político (la Monarquía) amplía sus actividades, en materia de obras públicas, militar, sanidad, auge de la burocracia. La originalidad del siglo XVII es que vincula la pobreza con los problemas globales de la sociedad española. Se la considera como una consecuencia más del gobierno y, por tanto, también con responsabilidad pública. La solución del problema pasa, por tanto, por resolver los problemas colectivos.

Pensadores como Sancho de Moncada, Jerónimo de Ceballos, López de Dexa, Fernández Navarrete y tantos otros, tratarán de arbitrar remedios eficaces para resolver las causas de esos males de la Monarquía.

En el XVIII, el período ilustrado es una fase de intensa reforma y modernización en España. Se pone de manifiesto en la mejora de ciudades, impulso a las artes, a la educación y las Universidades, a la racionalización de la vida colectiva. Se limitan las competencias de la Inquisición; se fomenta la dinámica colectiva con la protección de las Sociedades Económicas de Amigos del País, etc. En este contexto surge - después de la Caridad y la Beneficencia - la tercera etapa: la Asistencia Social. Se trata de una sistema público organizado de Servicios e Instituciones Sociales, destinado a la ayuda a personas y grupos en situación de necesidad. Supone el intento de superar la simple acción coyuntural, de cada caso concreto - propia de la beneficencia-, y va dirigida a la atención de necesidades básicas y no de la mera indigencia (4).

Por otro lado, los Ilustrados inician la desamortizacion de bienes de la Iglesia y de los municipios. Esto va a producir una importante consecuencia: favorecerá, desde entonces, la creciente intervención del Estado y la pérdida del protagonismo de la Iglesia.

En el reinado de Carlos III se pueden contemplar la diversidad de estrategias empleadas por los ilustrados en relación a la acción social. Actuaron, en efecto, en tres direcciones complementarias: Con una estrategia de prevención, mediante el impulso de Montepíos; con una orientación asistencial, mediante las Diputaciones de Barrio; y con centros de corrección, con establecimientos como el Hospicio de San Fernando.

A finales del siglo XVIII se producen dos acontecimientos históricos que influyen decisivamente en la respuesta pública ante los problemas sociales. Nos referimos a la revolución industrial y la revolución política. 
En España, ya en el XIX, coexisten la beneficencia y la asistencia social. Como consecuencia de las Cortes de Cádiz, se aprueba la Ley de Beneficencia de 1822 y, posteriormente, la de 1849. Pero en la segunda mitad del XIX, se deja notar el influjo del movimiento obrero, el socialismo, las medidas bismarkianas de previsión social, el socialismo fabiano en Inglaterra, la aparición de los partidos políticos, los movimientos sindicales y la cuestión social va a convertirse en asunto central de este siglo. En este contexto, se acelera el intervencionismo estatal en materia social (5).

En España la prueba de ello es la creación de la Comisión de Reformas Sociales en 1883, en el reinado de Alfonso XII. La Comisión tenía por objeto el estudio de todas las cuestiones relativas a los problemas sociales. Como resultado de su labor, se van a promulgar numerosas disposiciones en materia social. La Comisión fue el germen de la legislación social y asistencial posterior.

Ya a comienzos del siglo XX, se creará el Instituto de Reformas Sociales, en 1903, y el Instituto Nacional de Previsión. Durante la primera mitad de este siglo, comienzan los Seguros Sociales y la posterior aparición de la Seguridad Social como sistema público organizado de protección social. Por tanto coexisten en España en esta época, la Beneficencia, la asistencia social, los Seguros Sociales y la Seguridad Social.

El intervencionismo estatal adquiere nuevo vigor, como consecuencia de las dos guerras mundiales. Desencadena una dinámica de internacionalización de la cuestión social. Y ello porque afianza la creencia de que una paz duradera no se logra sólo resolviendo los problemas internacionales, sino los problemas sociales internos. Esta convicción dará lugar a la creación de organismos internacionales, como la OIT, que harán un gran esfuerzo para la homogeneización de la legislación social. El Estado del bienestar aparecerá también después de la Segunda Guerra Mundial, otorgando un papel creciente a los Servicios Sociales, pero orientados ya hacia la prevención y a la calidad de vida.

Entre tanto, durante el franquismo, en España hubo abundante legislación social. Pero, en lo que se refiere a esta materia, con gran dispersión administrativa, con una actitud paternalista y coexistiendo diferentes sistemas de prestación de asistencia. Por ello, el régimen de Franco supuso el retraso en la implantación de los Servicios Sociales en España respecto a otros países.

\section{LOS SERVICIOS SOCIALES EN LA ACTUALIDAD}

¿Y cuál ha sido el desarrollo de los Servicios Sociales a partir de la Constitución de 1978? La Constitución, en efecto, es decisiva, tan- 
to por las medidas políticas y jurídicas que introduce, como por las dinámicas sociales y prácticas materiales que desencadena, impulsa y favorece (6).

De hecho, la Constitución de 1978 no formula, expresamente, el reconocimiento del sistema público de Servicios Sociales. Pero esto no significa ninguna limitación, puesto que la Constitución es un texto comprometido con las cuestiones sociales, con la consecución de mayor grado de justicia social.

De manera expresa la Constitución contiene un mandato expreso para que los poderes públicos realicen una función promocional del bienestar social; así el artículo $1 .^{\circ}$ establece que: «España se constituye en un Estado social y democrático de Derecho, que propugna como valores superiores de su ordenamiento jurídico la libertad, la justicia, la igualdad y el pluralismo político».

El artículo 9.2 por su parte dice: «Corresponde a los poderes públicos promover las condiciones para que la libertad y la igualdad del individuo y de los grupos en que se integra sean reales y efectivas; remover los obstáculos que impidan o dificulten su plenitud y facilitar la participación de todos los ciudadanos en la vida política, económica, cultural y social».

Partiendo de una interpretación integradora de artículos como el $1 .^{\circ}, 9^{\circ}, 10$ y 14 , en relación con el Capítulo III, del Título I, principios rectores de la política social y económica, presta atención a determinados colectivos, como juventud (art. 48); disminuidos físicos, psíquicos y sensoriales (art. 49); tercera edad (art. 50) y a la familia e hijos (art. 39). De todo ello se desprende que sin los Servicios Sociales estos principios fundamentales quedarían vulnerados (7).

Los Servicios Sociales son una vía para tratar de cubrir los desajustes económicos y sociales y cuyo fin es conseguir la calidad de vida para todos y bienestar colectivo.

Otro punto importante a resaltar en la Constitución es su voluntad de aproximar los Servicios Sociales al ámbito geográfico donde se encuentran las necesidades. Buena prueba de ello es el artículo 148.20 donde se menciona, entre las competencias que pueden asumir las Comunidades Autónomas, la de «Asistencia Social».

Las dudas que inicialmente pudieron surgir al emplear la Constitución este concepto, fueron aclaradas al aprobarse con posterioridad los 17 Estatutos de Autonomía, ya que éstos asumen, aunque con diferentes denominaciones - asistencia social, bienestar social, servicios sociales- los Servicios Sociales. Mencionaré como ejemplo el Estatuto de Autonomía de Andalucía, cuyo artículo 13.22 afirma que la Comunidad Autónoma Andaluza tiene competencias exclusivas sobre asistencia y Servicios Sociales.

La Ley de Bases de Régimen Local (1985), refuerza la aproximación de los Servicios Sociales al ciudadano. Su artículo 25.2.k) esta- 
blece que el municipio ejercerá competencias en los términos de la legislación del Estado y de las Comunidades Autónomas en las siguientes materias: «Prestación de los Servicios Sociales y de promoción y reinserción social». El artículo 26.1.c) dice: «Los municipios con población superior a 20.000 habitantes deberán prestar en todo caso Servicios Sociales». Y por otra parte, el artículo 36 establece que «son competencias propias de la Diputación la prestación de servicios públicos de carácter supramunicipal, y en su caso supracomarcal».

Durante este período y a partir de 1982, comienzan a aprobarse en las Comunidades las leyes de Servicios Sociales.

Las 17 leyes de Servicios Sociales tienen por objeto implantar en las Comunidades Autónomas un sistema público de Servicios Sociales que ponga a disposición de las personas y grupos en que se integran, recursos, acciones, prestaciones para el logro de su pleno desarrollo. Así como la prevención, tratamiento y eliminación de las causas que conducen a la marginación.

Los principios inspiradores de los Servicios Sociales son hoy los siguientes:

1. Universalidad, atención a todos.

2. Igualdad, sin discriminación.

3. Solidaridad, justa distribución de recursos.

4. Prevención, eliminar causas de marginación.

5. Responsabilidad pública, recursos públicos.

6. Descentralización, acercar las instituciones más al ciudadano.

7. Normalización e Integración, cauces normales respetando el medio.

8. Participación, intervención de los ciudadanos en el control de los servicios sociales.

9. Planificación y Coordinación, equilibrio recursos y necesidades y armonización pública y privadas.

10. Globalidad, consideración total de la persona y de los grupos y no sólo de aspectos parciales.

\section{ORGANIZACION DE LOS SERVICIOS SOCIALES}

En la actualidad, los Servicios Sociales se encuentran organizados en Comunitarios y Especializados. Los Comunitarios constituyen la estructura básica del sistema público de servicios sociales (primer nivel de atención más próximo al usuario), siendo su finalidad el logro de unas mejores condiciones de vida mediante una atención integrada y polivalente.

Los Comunitarios se desarrollan en las Zonas de Trabajo Social, entendidas como demarcaciones para una prestación eficaz de los 
mismos (se estructuran en circunscripciones territoriales, de modo que haya cobertura total del territorio). Se ubican en los Centros de Servicios Sociales (8), donde se prestarán los Servicios siguientes:

1. Información, valoración, orientación y asesoramiento.

2. Cooperación social, impulso al asociacionismo, promoción de la vida comunitaria.

3. Ayuda a domicilio.

4. Convivencia y reinserción social (buscar alternativas al internamiento o remitir a los Servicios Sociales especializados).

Los Servicios Sociales especializados son aquellos que se dirigen hacia determinados sectores de población que por sus condiciones o circunstancias necesitan una atención específica (nivel secundario, más especializado técnicamente). Se dirigen a los siguientes sectores:

- Infancia, familia, juventud.

- Tercera edad.

- Mujer.

- Personas con deficiencias físicas, psíquicas o sensoriales.

- Toxicómanos y alcohólicos.

- Minorías étnicas, especialmente los gitanos.

- Grupos con conductas disociales (delincuencia, presos y ex reclusos).

- Colectivos marginados, pobreza y transeúntes.

- Otros colectivos (emigrantes, refugiados, asilados, inmigrantes), etc.

Para el desarrollo de estos servicios existen los siguientes centros especializados:

- Centros de Día.

- Centros de Acogida.

- Residencias.

- Guarderías.

- Centros Ocupacionales.

- Comunidades Terapéuticas.

- Albergues y Comedores, etc.

La financiación de los Servicios Sociales se hará con cargo a los Presupuestos públicos (Presupuestos Generales del Estado, Seguridad Social, Comunidades Autónomas y Corporaciones Locales). No obstante, está prevista la colaboración de la iniciativa privada así como la participación de los usuarios.

Las Leyes de Servicios Sociales regulan la distribución de competencias, reservándose las Administraciones Autonómicas las funciones de planificación, coordinación, supervisión y control, y las Corporaciones Locales la gestión, ejecución y desarrollo de los servicios, 
siendo por tanto muy importantes las funciones de los Ayuntamientos y Diputaciones en los servicios, como lugares más próximos al ciudadano (9).

Pero no en las Corporaciones Locales aisladamente sino incardinadas o coordinadas en la Comunidad Autónoma y el Estado, formando así una estructura, un conjunto o un sistema general y público de bienestar social, como la educación o la sanidad, tal como lo pone de manifiesto la aprobación del Plan Concertado para el desarrollo de prestaciones básicas de Servicios Sociales.

El marco legal básico habría que completarlo con la alusión a leyes generales que regulan sectores especializados:

- La LISMI.

- La Ley General Penitenciaria.

- La Ley de Refugiados y Asilados, etc.

Así como con la aprobación de planes integrales de actuación administrativa como:

- Plan Nacional contra la Droga.

- Plan para la igualdad de oportunidades de la mujer.

- Plan Nacional de desarrollo gitano.

- Plan gerontológico-geriátrico.

- Plan para la juventud, etc.

\section{EL MARCO ADMINISTRATIVO CENTRAL}

El hecho de la configuración como sistema público de los Servicios Sociales, se manifiesta por tanto en el marco normativo que hemos mencionado, con las prestaciones específicas que establece y los equipamientos propios. Pero también, correlativamente, por la existencia de un marco administrativo, que gestiona y organiza el funcionamiento del sistema.

En julio de 1988 se crea el Ministerio de Asuntos Sociales, que pasa a ser, en sustitución del Ministerio de Trabajo y Seguridad Social, el núcleo fundamental de los Servicios Sociales prestados por el Estado.

Este Ministerio tiene funciones como dirección y planificación de los Servicios Sociales, atendiendo a las áreas de bienestar social; cooperación con ONG, ejerciendo la tutela sobre entidades asistenciales ajenas a la administración - la ONCE o la Cruz Roja-, y el protectorado sobre fundaciones de beneficencia. Por último, la gestión de la asignación tributaria del IRPF para otros fines de interés social.

De este Ministerio dependen organismos fundamentales en lo que a Servicios Sociales se refiere (10): 
- Dirección General de Acción Social.

- Dirección General de Protección Jurídica del Menor.

- Secretaría General del Real Patronato de Prevención y atención a personas con minusvalía.

Están adscritos al Ministerio los organismos autónomos siguientes:

- Inserso.

- Instituto de la Mujer.

- Instituto de la Juventud.

El Ministro/a preside los órganos colectivos siguientes:

- Comisión Interministerial de la Juventud.

- Consejo del Protectorado del Estado sobre la ONCE.

- Presidencia efectiva del Real Patronato de Prevención y atención a las personas con minusvalía.

- Así como las relaciones con el Consejo de la Juventud de España.

Pese a la existencia del Ministerio de Asuntos Sociales, otras actividades relativas a los Servicios Sociales se encuentran en otros Ministerios, como:

- M. Trabajo (emigración).

- M. Justicia (Institución penitenciarias y CAS).

- M. Sanidad (Delegado Gobierno Plan Nacional de Drogas).

\section{CONSIDERACIONES FINALES}

Para finalizar, cabe reflexionar sobre diversos aspectos que inciden sobre los Servicios Sociales. Aunque se están consolidando en España, como un sistema público, son, no obstante, el resultado de una dilatada evolución histórica que ha estado sujeta a toda suerte de influencias. Factores históricos, políticos, económicos, religiosos, culturales, jurídicos - relacionados entre sí- han condicionado su trayectoria.

Los primeros esbozos de alguna responsabilidad pública ante situaciones de necesidad, se producen en torno al siglo XVI. Pero en esta época -y durante varios siglos todavía-, la respuesta se realiza como atención al caso concreto y a la situación particular. A menudo la forma de realizarlo que se empleaba era la institucionalización - es decir, el ingreso de la persona en algún establecimiento al efecto--. Pero estas instituciones generaban aún mayor segregación y marginación de los individuos.

El papel y la responsabilidad del Estado fue en aumento también como consecuencia de la desamortización. La falta de recursos de las fundaciones religiosas cuando, en el XIX, se incrementan las necesi- 
dades, obligan a la rápida sustitución de la beneficencia privada por la pública.

La cuestión social se encuentra en el substrato de la aparición de los Servicios Sociales. Provocó como respuesta, entre otros aspectos, el paulatino intervencionismo estatal. Esa intervención adopta, en un primer momento durante el XIX, una modalidad limitadora de los excesos mediante leyes sociales protectoras. Pero esa misma dinámica generará demandas de medidas preventivas, equipamientos y servicios que, con el tiempo, se estructurarán como Servicios Sociales.

$\mathrm{Si}$ en otros países europeos esa evolución fue más rápida, el franquismo en España retrasó el proceso. Aunque introduce numerosas medidas concretas de acción social, lo realizó con mentalidad paternalista y con gran dispersión organizativa. De ahí que en ese período de nuestra historia España no contara aún con un nivel de cobertura de los Servicios Sociales, semejante al disponible en otros países europeos.

El retraso del sistema de Servicios Sociales en España en comparación a otros países, se ha reducido a partir de la Constitución de 1978 y las dinámicas jurídicas y políticas que ocasionó. Con la Constitución los Servicios Sociales se dirigen a todos los ciudadanos, siguiendo el principio de universalidad, aunque en la práctica no todos los grupos sociales necesitados cuenten con cobertura para sus necesidades. La falta de recursos económicos, el incremento de demandas debido a la crisis económica, la reciente implantación, etc., origina que haya sectores sociales todavía desatendidos.

La tendencia actual se dirige a reforzar los Servicios Sociales comunitarios, como primer nivel de atención, y a la desinstitucionalización. De esta manera se logra mantener a la persona en su medio habitual, propiciando así la integración.

Tanto a nivel central como autonómico, se producen situaciones muy diferenciadas de acceso a los Servicios Sociales especializados. Existen, en el momento actual, colectivos bastante mejor protegidos - p. ej. tercera edad o toxicómanos-, que otros - como gitanoscon menores dotaciones y servicios disponibles.

La iniciativa social ha desempeñado un papel relevante en la atención a las necesidades sociales. La legislación vigente apoya a las instituciones del voluntariado, y es creciente su protagonismo en la prestación de Servicios Sociales. Esta política se encuentra igualmente avalada por directrices de la propia Comunidad Económica Europea.

En España, desde la transición política, las distintas Administraciones - Central, Autonómica, Local - han realizado importantes esfuerzos en la consolidación de los Servicios Sociales, cada una dentro del ámbito de sus competencias. Pero resultan todavía insuficientes. Además, dado el retraso del que se partía, gran parte de los 
recursos se han orientado a la creación y puesta en marcha tanto de las estructuras administrativas como de centros y equipamientos. Por ello, pese a los esfuerzos que se realizan, puede dar la impresión que los recursos no llegan a los verdaderos necesitados.

La evolución jurídica, política y administrativa y las prácticas materiales se encaminan a la configuración de los Servicios Sociales como un elemento más para elevar la calidad de vida, junto al sector educativo, al de empleo, salud, seguridad social, etc. Los Servicios Sociales hoy cuentan - al igual que estos otros sectores- con normativa propia, ámbito de actuación diferenciado, prestaciones específicas, estructura y equipamientos propios, con financiación pública, etc. Por ello los Servicios Sociales quedan configurados como un sistema público.

\section{BIBLIOGRAFIA}

1. Casado, D. y Guillén, E.: Introducción a los Servicios Sociales. Ed. Acebo, Madrid, 1987.

2. El esquema que fue señalado por Las Heras, P. y Cortajarena, E.: Introducción al Bienestar Social. Ed. Federación Española Asistentes Sociales, Madrid, 1984. También se realiza un importante recorrido histórico en VV.AA.: Cuatro siglos de Acción Social. De la Beneficencia al Bienestar Social. Ed. Siglo XXI, Madrid, 1985. Y en VV.AA.: Historia de la Acción Social Pública en España. Ed. Siglo XXI, Madrid, 1990.

3. Rubio Nombela, G.: «Caridad, beneficencia y asistencia social». En VV.AA. Problemas fundamentales de beneficencia y asistencia social. Ed. Ministerio de Gobernación, Madrid, 1967.

4. Martín Mateo, R.: «Sobre el concepto de Asistencia Social. En VV.AA. Problemas fundamentales de beneficincia y asistencia social. Ed. Ministerio de Gobernación, Madrid, 1967.

5. Alemán Bracho, C.: El sistema público de Servicios Sociales en España. Ed. Impredisur, Granada, 1991.

6. Un análisis interesante del desarrollo constitucional lo realizó Aznar López, M.: «Los Servicios Sociales: Marco Constitucional en España». En VV.AA. ¿Bienestar Social en España?, Ed. INTRES, Barcelona, 1989.

7. VV.AA.: Cambio social y Servicios Sociales. Ed. Secretaría Federal del PSOE, Madrid, 1981.

8. García, G.: Los centros de Servicios Sociales. Conceptualización y desarrollo operativo. Ed. Siglo XXI, Madrid, 1988.

9. Perdomo, J. G.: Servicios Sociales. Leyes Autonómicas. Ed. Ministerio Servicios Sociales, Madrid, 1989.

10. Como instrumento de actualización en estos puntos es interesante la utilización de los Boletines de Documentación del CEDISS y el Repertorio Legislativo de Acción Social, editados por el Ministerio de Asuntos Sociales. 